\title{
Gram-Negative Bacteria Bloodstream Infections in Patients with Hematological Malignancies - The Impact of Pathogen Type and Patterns of Antibiotic Resistance: A Retrospective Cohort Study
}

\author{
Yishu Tang' \\ Cong $\mathrm{Xu}^{2}$ \\ Han Xiao ${ }^{2}$ \\ Liwen Wang ${ }^{2}$ \\ Qian Cheng ${ }^{2}$ \\ $\mathrm{Xin} \mathrm{Li}^{2}$
}

'Department of Emergency, The Third Xiangya Hospital, Central South University, Changsha, Hunan, People's Republic of China; ${ }^{2}$ Department of Hematology, The Third Xiangya Hospital, Central South University, Changsha, Hunan, People's Republic of China
Correspondence: Xin $\mathrm{Li}$ Department of Hematology, The Third Xiangya Hospital, Central South University, Changsha, 410013, Hunan,

People's Republic of China

Tel +86-73|-886|88|4 (886/82|4)

Email lixiner1975@I63.com
Background: Enterobacteriaceae (EB) and non-fermentative bacteria (NFB) are the main pathogens responsible for gram-negative bloodstream infections (GN-BSI) in patients with hematological malignancies (HMs). These two pathogen types have heterogeneous resistance mechanisms to antibiotics. However, the impact of pathogen species and pattern of antibiotic resistance on the outcomes of patients with HMs remains unclear.

Methods: We retrospectively collected clinical data of patients with HMs at three comprehensive hospitals in Hunan Province, China, between January 2010 and May 2018. The data analyzed the impact that different species and patterns of antibiotic resistance had on the outcome of patients with HMs.

Results: The majority of the 835 monomicrobial isolates collected from patients with HMs and GN-BSIs were Enterobacteriaceae (75.7\%). While detections of MDR pathogens in BSIs as a whole are decreasing, sub-analysis shows that detections of extended spectrum $\beta$ lactamase-producing (ESBL) Enterobacteriaceae and carbapenem-resistant pathogens in BISs are rising. Comparing different species, the early mortality rate associated with infections caused by NFB was significantly higher than infections caused by Enterobacteriaceae (22.6\% vs 9.7\%, p < 0.001). Across different multidrug-resistant (MDR) patterns, ESBL bacteria did not have a significant impact on health outcomes. Carbapenem-resistant bacteria, on the other hand, were observed to significantly affect early mortality rate, such as carbapenem-resistant Klebsiella pneumoniae $(36.0 \%$ vs $7.6 \%, \mathrm{P}<0.001)$ and carbapenemresistant non-fermentative bacteria $(44.7 \%$ vs $16.5 \%, \mathrm{P}<0.001)$.

Conclusion: Our findings suggest that both species and patterns of antibiotic resistance can affect the early mortality of patients with HMs during BSI.

Keywords: multidrug-resistant patterns, hematological malignancies, gram-negative bloodstream infections, carbapenem-resistant bacteria

\section{Introduction}

In the past few decades, gram-negative bacteria (GNB) have become the main pathogens responsible for bloodstream infections (BSI) in patients with hematological malignancies (HMs), accounting for $50-75 \%$ of all BSI cases. ${ }^{1-4}$ GNB are mainly composed of Enterobacteriaceae (ie, Escherichia coli, Klebsiella pneumoniae, Enterobacter cloacae, and non-fermentative bacteria (NFB) (ie, Pseudomonas aeruginosa, Acinetobacter baumannii, Stenotrophomonas maltophilia) with 
associated infections resulting in mortality rates ranging from $15 \%$ to $42 \% .^{3-8}$ Although the use of broad-spectrum antibiotics and appropriate administration of antimicrobial therapies has led to a decrease in patient mortality, at the same time, the proportion of multidrug-resistant (MDR) bacteria has steadily increased as an unfortunate consequence.

MDR is defined as acquired non-susceptibility to at least one agent in three or more antimicrobial categories. ${ }^{9}$ MDR pathogens can display increased resistance to clinical antibiotics and may result in treatment failure. Among multidrug-resistant GNB, Enterobacteriaceae with an extended spectrum $\beta$ lactamase (ESBL) phenotype and carbapenem-resistant (CRE) isolates in particular have become an increasing concern in the medical and health sectors. ${ }^{8,10}$ Patients with HMs who have disease-related immunosuppression and long-term exposure to broad-spectrum antibiotics are especially at risk. ${ }^{34}$

Whether pathogen type or patterns of antibiotic resistance affect the prognosis of patients with HMs remains debatable. ${ }^{3,8,10-12}$ Our previous studies showed that endogenous (host-related factors, such as disease status, organ functions, and nutritional status) factors or exogenous (treatment-related factors, such as inappropriate initial antimicrobial therapy (IIAT)) factors had impact on patient prognoses. ${ }^{13,14}$ However, bacterial-related factors such as pathogen species or patterns of antibiotic resistance may also act as risk factors leading to poorer health outcomes in HM patients, but prior literature focusing on these bacterial-related factors is limited and inconsistent. One study showed that the 21-day prognosis of multidrugresistant gram-negative bacteria (MDR-GN) BSI was worse compared to BSIs of drug-sensitive gram-negative bacilli, ${ }^{8,10}$ while other studies have shown opposing findings. ${ }^{11,12}$ In addition, the influence of bacterial pathogen type in BSI on prognosis of patients is also an area requiring further research. Studies have shown that the prognosis of patients with non-fermentative bacterial BSIs was worse compared to Enterobacteriaceae induced BSIs, emphasizing the heterogeneity of different pathogens in GNB-BSI. ${ }^{13,14}$ Given the state of current literature, there is a need to increase our understanding of whether BSI pathogen type or patterns of antibiotic resistance can affect the outcomes of HM patients, which can hold important clinical implications and inform policies concerning antimicrobial stewardship and infection control surveillance.
In this study, we retrospectively analyzed multi-center clinical data of patients with HMs complicated with GNBBSI over a 9 year timeframe, with the purpose of exploring the influence of different pathogen type and antibiotic resistance patterns on prognosis of patients.

\section{Patients and Methods \\ Setting and Study Design}

We identified all episodes of GN-BSIs in patients (age $\geq 16$ years) with hematologic malignancies at three universityaffiliated hospitals in Hunan Province, China, from January 2010 to May 2018. The following characteristics were collected for each patient: demographic information, malignancy characteristics, comorbidities, laboratory data, antibiotic agents, and the outcome of infection. For each bacterial isolate, the antimicrobial susceptibility was determined and analyzed. In patients who had multiple positive cultures with the same specificity and sensitivity, only the first culture was included for analysis. Blood culture samples which detected different bacterial strains within $48 \mathrm{~h}$ were defined as polymicrobial bacteremia and excluded from this study due to the limited sample size and possible confounding effects. Anti-infection therapies were performed according to pre-defined guidelines. ${ }^{6,15}$ The primary outcome was all-cause mortality within 7-days after BSI onset.

\section{Definitions}

The following terms were defined before data analysis. BSI was defined by the isolation of infectious organisms from blood culture specimens in patients with compatible clinical signs and symptoms. ${ }^{16}$ Neutropenia and profound neutropenia were defined as an absolute neutrophil count (ANC) of $<500$ cells $/ \mathrm{mm} 3$ and $<100$ cells $/ \mathrm{mm} 3$, respectively. ${ }^{15}$ Pitt bacteremia score was calculated at the time of fever onset. ${ }^{17}$ The date of BSI onset was represented by the collection date of the first positive blood culture (index culture). BSIs were classified as nosocomial if the index blood culture was drawn more than $48 \mathrm{~h}$ after hospital admission. ${ }^{6}$ MDR was defined as nonsusceptibility to at least one agent in three or more antimicrobial categories. ${ }^{9}$

Carbapenem-resistant Enterobacteriaceae (CRE) was defined as Enterobacteriaceae isolates demonstrating resistance to any carbapenem (ertapenem, meropenem, imipenem, and/or doripenem), based on antimicrobial susceptibility testing (AST). ${ }^{18}$ Disease status was assessed by 
the most recently available bone marrow biopsy and categorized as remission, relapsed, or uncontrolled malignancy, as previously defined. ${ }^{19}$ According to our population characteristics and cutoff value, sustained neutropenia exceeding 21 days was defined as prolonged neutropenia. Acute respiratory failure and acute renal failure were described in Tang et al. ${ }^{19}$ Antibiotic exposure was defined as any antimicrobial therapy lasting more than $48 \mathrm{~h}$ in the previous one month. ${ }^{20}$ Inappropriate initial antimicrobial therapy (IIAT) refers to antibiotic regimens prescribed and administered during the first $72 \mathrm{~h}$ after suspecting BSI, and was not active against the pathogen identified by culture and in vitro susceptibility testing. ${ }^{19,21}$

\section{Antibiotic Susceptibility Test}

Bloodstream isolate identification and antibiotic susceptibility tests were performed on VITEK 2 Compact (bioMe'rieux SA, Marcy l'Etoile, France). VITEK 2 Compact was used to screen ESBL positive E. coli or $K$. pneumoniae. According to the CLSI guidelines, phenotypic confirmatory test for ESBL was performed using both disk containing $30 \mu \mathrm{g}$ ceftazidime and disk containing $30 \mu \mathrm{g}$ cefotaxime, alone and in combination with $10 \mu \mathrm{g}$ clavulanic acid (Becton-Dickinson, Franklin Lakes, NJ, USA). Strains producing ESBL were confirmed as a $\geq 5 \mathrm{~mm}$ increment in a zone diameter for either combination treatment versus corresponding monotreatment. CRE was defined as Enterobacteriaceae isolates demonstrating resistance to any carbapenem (ertapenem, meropenem, imipenem, and/or doripenem), based on antimicrobial susceptibility testing (AST). Carbapenem resistance was defined as an ertapenem $\mathrm{MIC} \geq 2 \mu \mathrm{g} / \mathrm{mL}$ and meropenem and/or imipenem MIC $\geq 4 \mu \mathrm{g} / \mathrm{mL}^{18}$

\section{Statistical Analysis}

Statistical analysis was performed with SPSS version 19.0 for Windows. Categorical variables were compared using chi square tests. Variables with $P \leq 0.1$ (two tailed) in the bivariate analysis were taken as candidates for multivariate analysis. Logistic regression was used for multivariate analysis to identify independent risk factors for 7-day mortality. Cutoff value means the diagnostic threshold, it represents the clinical decision point. The cutoff values for continuous variables were set according to clinical practice or laboratory references by using the receiver operating characteristic curve (ROC). In cases where less than 5\% of data were missing, missing values for continuous variables were replaced via mean imputation; missing categorical variable values were replaced via mode imputation. All $\mathrm{p}$ values were two sided, and $p \leq 0.05$ was considered significant.

\section{Results}

\section{Pathogens and the Trend of Antibiotics Resistance Over the Years}

In the present study, a total of 835 strains of GNB were detected, 633 strains (75.7\%) were Enterobacteriaceae bacteria and 177 strains $(21.2 \%)$ were non-fermentative bacteria. The majority (53.6\%) of Enterobacteriaceae bacteria were Escherichia coli, while the majority (61.6\%) of non-fermentative bacteria consisted of Pseudomonas aeruginosa (Table 1).

Broadly speaking, the proportion of BSI attributable to MDR-GNB showed a downward trend from $75.7 \%$ in 2010 2011 to $63.0 \%$ in 2016-2018 (Figure 1A, refer to Supplementary Table 1and 1a). Sub-analysis also revealed that the proportion of MDR Enterobacteriaceae in all Enterobacteriaceae decreased over the years; however, the MDR rates of non-fermentative bacteria in all nonfermentative bacteria showed an upward tendency with detection rates of non-fermentative bacteria increasing from $46.7 \%$ in 2010-2012 to 79.5\% in 2016-2018 (Figure1B, refer to Supplementary Table 1 and $\underline{1 b}$ ). ESBL-producing rate increased from $31.1 \%$ in $2010-2014$ to $50.5 \%$ (Figure $1 \mathrm{C}$, Supplementary Table 1 and $\underline{1 c)}$ in 2015-2018 in Enterobacteriaceae. The percentage of carbapenem-resistant strains in both Enterobacteriaceae and GN-bacteria increased significantly, from $0.0 \%$ in 2010 to $13.1 \%$ in 2018 among Enterobacteriaceae and from $3.0 \%$ in 2010 to $15.8 \%$ in 2018 among GN-bacteria (excluding Stenotrophomonas maltophilia) (Figure 1D, Supplementary Table 1 and 1d).

Table I Composition of GN-Bacteria Isolated from Bloodstream Infection in Patients with HMs

\begin{tabular}{|l|l|}
\hline GN-Bacteria & $\mathbf{n = 8 3 5}$ \\
\hline Enterobacteriaceae & $632(75.7 \%)$ \\
Escherichia coli & $339(53.6 \%)$ \\
Klebsiella pneumoniae & $197(31.2 \%)$ \\
Enterobacter cloacae & $33(5.2 \%)$ \\
Others Enterobacteriaceae & $63(10.0 \%)$ \\
\hline Non-fermentative bacteria & $177(21.2 \%)$ \\
Pseudomonas aeruginosa & $109(61.6 \%)$ \\
Acinetobacter baumannii & $25(14.1 \%)$ \\
Stenotrophomonas maltophilia & $20(11.3 \%)$ \\
Other Non-fermentative bacteria & $23(13.0 \%)$ \\
\hline Other GN-bacteria & $26(3.1 \%)$ \\
\hline
\end{tabular}



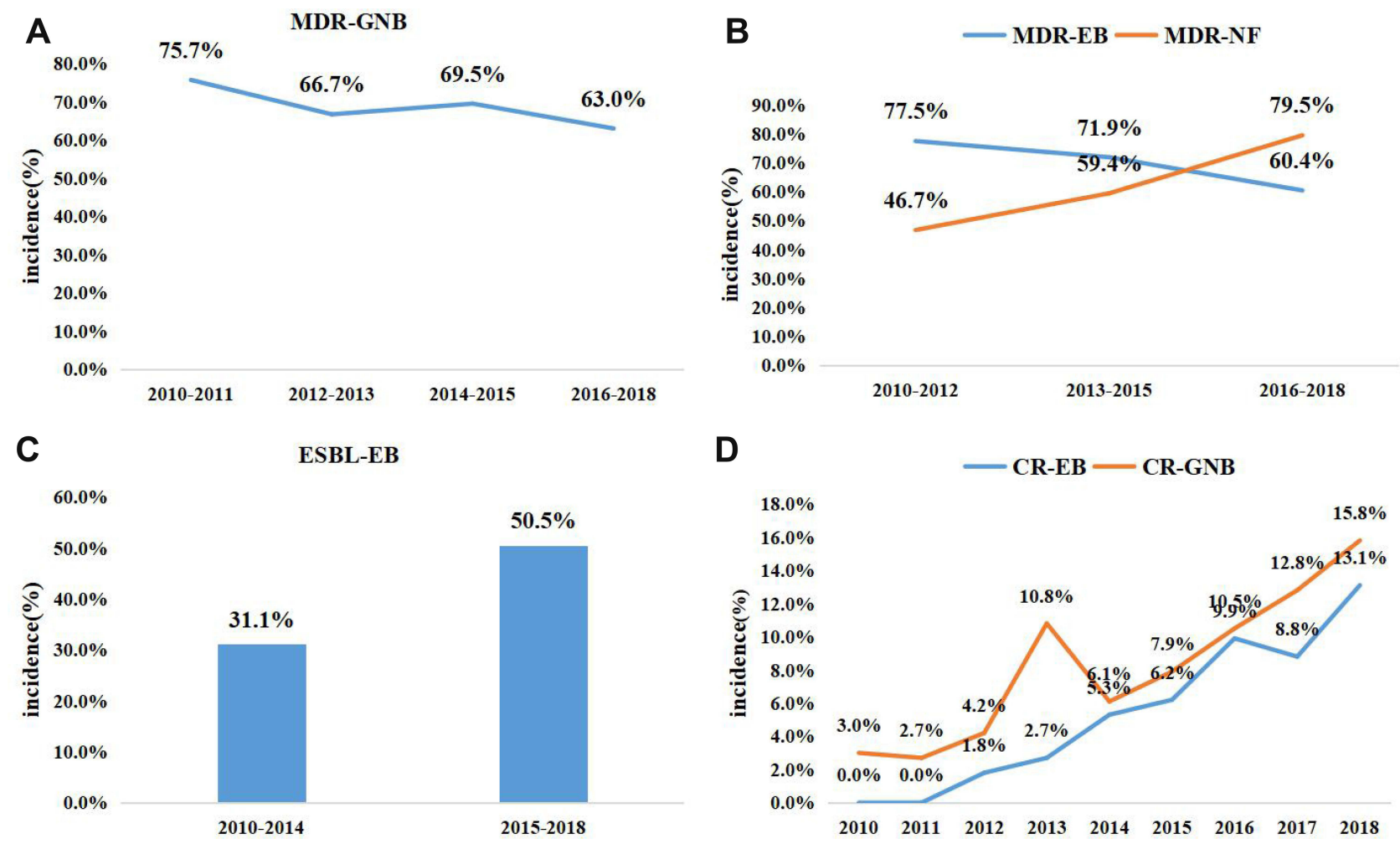

Figure I Proportion of BSI based on pathogen resistance phenotype from 2010-2018. The X-axes represents years. (A) The change in percentages of MDR detection rate in all GN-bacteria over the study period. (B) The change in percentages of MDR detection rate in EB and NF isolates over the study period. (C) The change in the detection rate of ESBL producing Enterobacteriaceae. (D) The change in carbapenem-resistant bacteria detection rate from Jan 2010-May 2018 in EB and NF strains.

Abbreviations: MDR-GNB, multidrug resistance-gram negative bacteria; MDR-EB, multidrug resistance -Enterobacteriaceae; MDR-NF, multidrug resistance-non fermentative bacteria; ESBL-EB, extended-spectrum $\beta$-lactamases producing-Enterobacteriaceae; CR-EB, carbapenem resistant-Enterobacteriaceae; CR-GNB carbapenem resistantGram negative bacteria.

\section{Impact of Pathogen Type on Mortality- Non-Fermentative Bacterial BSI is an Independent Risk Factor for Early Mortality} In terms of bacterial species, the early mortality rate of patients with non-fermentative bacteria associated BSIs was significantly higher than that of patients with Enterobacteriaceae associated BSIs $(22.6 \%$ vs 9.7\%, 2.733 (1.760-4.244), $\mathrm{p}<0.001$ ) (Figure 2A and Supplementary Table 2). BSI due to Acinetobacter baumannii demonstrated the highest early mortality rate (64.0\%, 16/25), followed by infections resulting from Stenotrophomonas maltophilia $(35.0 \%, 7 / 20)$ and Pseudomonas aeruginosa $(13.8 \%, 15 / 109)$ (Figure 2B and Supplementary Table 2). The early mortality rate of infections due to Escherichia coli and Klebsiella pneumoniae was similar (11.2\% vs $11.2 \%$ ) (Figure 2).

A multivariate analysis was conducted to determine whether pathogen type affected the prognosis of patients. Results show that non-fermentative bacteria BSI is an independent risk factor for the 7-day mortality of patients
(Tables 2, 2.093 (1.077-4.067), $\mathrm{p}=0.029)$. Other patient characteristics which contributed to worse mortality outcomes were disease state, presence of acute respiratory failure, use of vasopressors, and inadequate treatment (Table 2).

Table 3 shows demographic and clinical characteristic differences between Enterobacteriaceae BSI patients and non-fermentative bacteria BSI patients. The incidence of respiratory failure, rate of urine tube placement, and 72 $\mathrm{h}$ IIAT are significantly higher in the non-fermentative bacteria BSI patient group compared to the Enterobacteriaceae BSI patient group. Most other variables (such as age, gender, disease status, etc.) showed no significant differences.

\section{Patterns of Antibiotics Resistance Impact Mortality-BSI Associated with Carbapenem Resistant GN-Bacteria Has Poorer Prognosis for Patients}

We did not find a significant association between MDR pathogens and patient prognosis in our study. Additionally, 

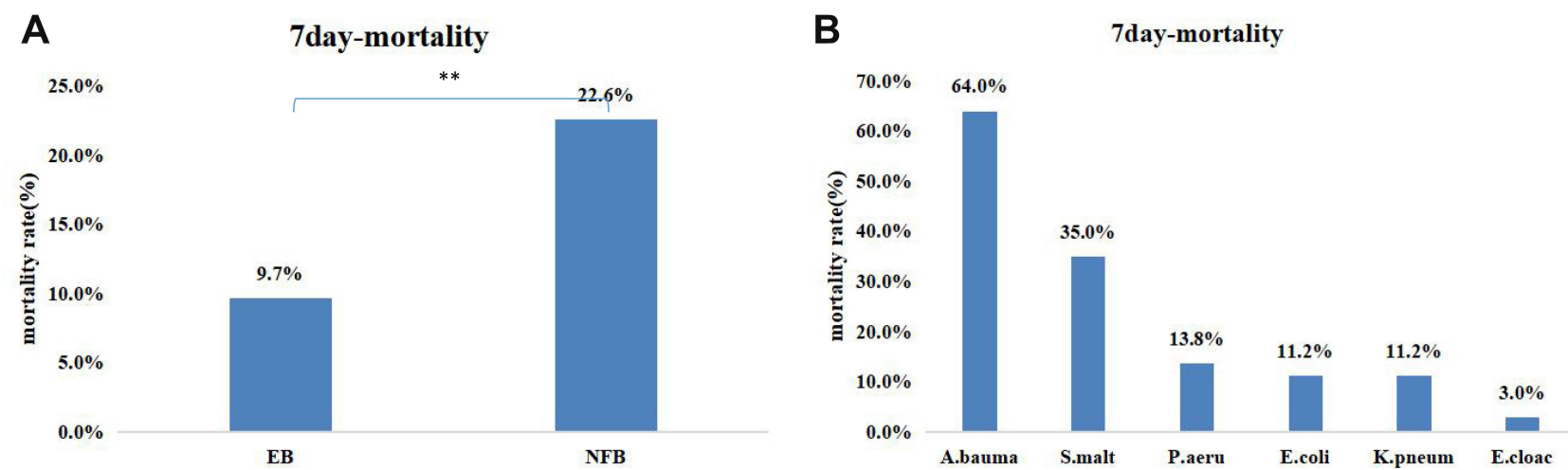

Figure 27 day mortality rate of patients with BSI: Enterobacteriaceae vs non-fermenting bacteria BSI. (A) 7 day mortality rate of Enterobacteriaceae and Non fermentative bacteria; (B) 7 day mortality rate of different strains. $* * \mathrm{P}<0.001$.

Abbreviations: EB, Enterobacteriaceae; NFB, non fermentative bacteria; A. bauma, Acinetobacter baumannii; S. malt, Stenotrophomonas maltophilia; P. aeru, Pseudomonas aeruginosa; E. coli, Escherichia coli; K.pneum, Klebsiella pneumoniae; E. cloac, Enterobacter cloacae.

ESBL-production isolates had no significant impact on the early prognosis of patients with $\mathrm{HMs}(\mathrm{P}>0.05)$ (Figure 3A and B, Supplementary Table 3). In Carbapenem-resistance Gram-negative strains, the early mortality of patients was significantly higher than carbapenem-sensitive strains, especially in carbapenem-resistant Klebsiella pneumoniae (CR-KP) strains (36.0\% vs $7.6 \%$, 6.880 (2.548-18.576), $\mathrm{P}<0.001)$ and non-fermentative bacteria $(44.7 \%$ vs $16.5 \%, \mathrm{P}<0.001)$ (Figure $3 \mathrm{C}$ and $\mathrm{D}$, Supplementary Table 3).

\section{Discussion}

Our study data show that both pathogen type and pattern of antibiotic resistance can affect early outcomes of HM patients with GNB-BSI. To our knowledge, this is the first study evaluating the effect of bacteriological factors on the prognosis of patients with HMs. These findings have important implications for managing the increasingly common problem of bacteremia associated with nonfermentative and carbapenem-resistant bacteria in immunocompromised patients.

Previous studies conducted in patients with HMs have shown that Enterobacter are responsible for 55-70\% of GN-bacteria BSI in patients with HMs, while nonfermenting bacteria are responsible for $20-40 \%$ of BSI worldwide. $^{2,22}$ In comparison, the rate of Enterobacter related BSI is reported to be about $70-75 \%$ in China, 4,7,23 consistent with our present study. Although the overall prognosis of patients with bacterial infections has improved with the continuous administration of broadspectrum antibiotics, antibiotic resistance has also become progressively more severe. According to the United States Centers for Disease Control and Prevention (CDC), antibiotic resistant GN-bacteria is becoming increasingly widespread, and the prevalence of BSI associated with carbapenem-resistant GN-bacteria is also rising over time; the prevalence of CR-KP BSI was lower than $1 \%$ in 2000 but has since grown to $21 \%$ in $2018 .^{24}$ This upward pattern has also been identified in other countries. $^{24,25}$ Our study identifies a similar trend gathered from 9-years of data. Carbapenem-resistant Enterobacter related BSIs that were not detected in 2010 have increased to a prevalence of $13.1 \%$ in 2018 .

It is well documented that the type of pathogenic bacteria can affect the prognoses of patients with HMs suffering from BSI. A study surveying 575 patients with HMs revealed that the 21-day mortality rate of patients with GN-BSI was significantly higher than the mortality rate of patients with bacteremia associated with Grampositive bacteria $\left(16.9 \%\right.$ vs $5.6 \%$, p < 0.001). ${ }^{3}$ Among different GN-bacteria species, the mortality of patients with Pneumocystis, Aeruginosa, and Baumannii related BSI was remarkably higher than that of patients with other pathogen related BSIs $(p<0.05)$, ${ }^{1,3,26,27}$ suggesting the heterogeneity of bacteria could influence the prognosis of patients. In our present study, the patients with BSI caused by non-fermentative bacteria had a significantly higher early mortality rate compared to patients with Enterobacter caused BSI (22.6\% vs 9.7\%, $\mathrm{p}<0.001)$, with Acinetobacter baumannii- associated BSI patients having the highest mortality rate $(64.0 \%)$. Furthermore, the incidence of respiratory failure, rates of urinary catheter placement, and $72 \mathrm{~h}$ IIAT were significantly higher in patients with non-fermentative bacteria BSI compared to patients with Enterobacteriaceae BSI, demonstrating the need for prudent administration 


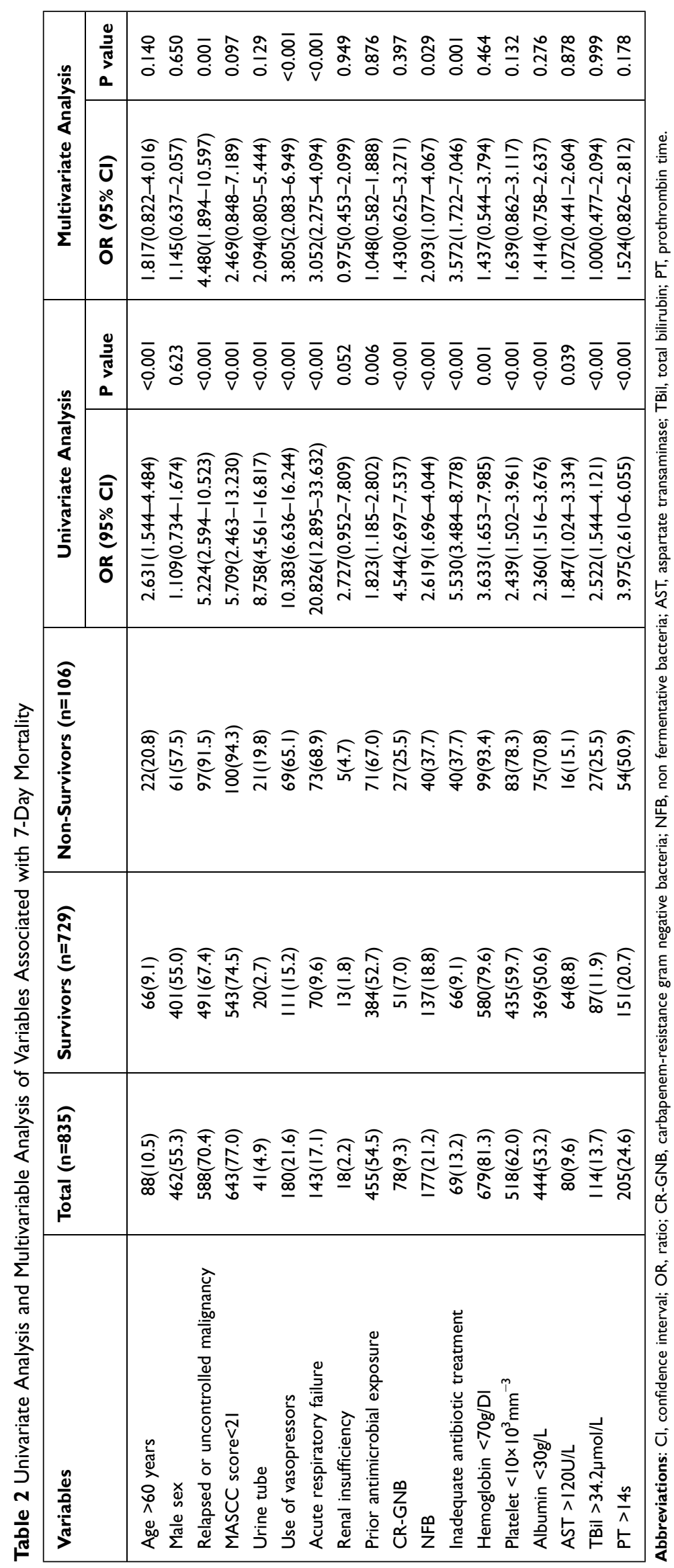


Table 3 Demographic and Clinical Characteristics of EB and NF Associated BSI Patients

\begin{tabular}{|c|c|c|c|c|}
\hline Variables & $E B(\%) n=632$ & NF (\%) $n=177$ & OR (95\% Cl) & $\mathbf{P}$ \\
\hline \multicolumn{5}{|l|}{ Demographic information } \\
\hline Age $>60$ years & $64(10.1)$ & $22(12.4)$ & $1.260(0.752-2.110)$ & 0.380 \\
\hline Male sex & $349(55.2)$ & $96(54.2)$ & $0.96 \mid(0.688-1.343)$ & 0.816 \\
\hline \multicolumn{5}{|l|}{ Underlying malignancies } \\
\hline Acute myeloid leukemia & $315(49.8)$ & $91(51.4)$ & $1.065(0.763-1.486)$ & 0.712 \\
\hline Acute lymphoblastic leukemia & $223(35.3)$ & $55(35.1)$ & $0.827(0.578-1.182)$ & 0.297 \\
\hline Lymphoma & $38(6.0)$ & $12(6.8)$ & $\mathrm{I} .137(0.58 \mathrm{I}-2.225)$ & 0.708 \\
\hline \multicolumn{5}{|l|}{ Disease status } \\
\hline Remission & $188(29.7)$ & $54(30.5)$ & $1.037(0.722-1.490)$ & 0.845 \\
\hline Relapsed or uncontrolled & $444(70.3)$ & $123(69.5)$ & $0.964(0.67 \mid-1.386)$ & 0.845 \\
\hline \multicolumn{5}{|l|}{ Risk factors } \\
\hline Charlson Comorbidity index $\geq 4$ & $103(16.3)$ & $32(18.1)$ & $1.133(0.732-1.755)$ & 0.574 \\
\hline Pitt bacteremia score $\geq 4$ & $153(24.2)$ & $45(25.4)$ & $1.067(0.727-1.567)$ & 0.740 \\
\hline MASCC score $<21$ & $491(77.7)$ & $134(75.7)$ & $0.895(0.605-1.323)$ & 0.578 \\
\hline \multicolumn{5}{|l|}{ Neutropenia } \\
\hline Profound neutropenia & $556(88.0)$ & $148(83.6)$ & $0.698(0.438-1.110)$ & 0.127 \\
\hline Prolonged neutropenia & $221(35.0)$ & $49(27.7)$ & $0.712(0.493-1.028)$ & 0.069 \\
\hline Previous chemotherapeutics & $575(91.0)$ & $152(85.9)$ & $0.603(0.364-0.997)$ & 0.047 \\
\hline Urine tube & $22(3.5)$ & $18(10.2)$ & $3.139(1.644-5.994)$ & $<0.001$ \\
\hline CVC & $258(40.8)$ & $78(44.1)$ & $1.142(0.816-1.599)$ & 0.439 \\
\hline \multicolumn{5}{|l|}{ Dysfunctional organ systems } \\
\hline Use of vasopressors & $130(20.6)$ & $45(25.4)$ & $1.316(0.892-1.943)$ & 0.166 \\
\hline Acute respiratory failure & $95(15.0)$ & $44(24.9)$ & $1.870(1.248-2.803)$ & 0.002 \\
\hline Renal insufficiency & $\mathrm{II}(\mathrm{I} .7)$ & $7(4.0)$ & $2.325(0.888-6.088)$ & 0.078 \\
\hline \multicolumn{5}{|l|}{ Antibiotic therapy } \\
\hline Prior antimicrobial exposure & $353(55.9)$ & $91(5 \mid .4)$ & $0.836(0.599-1.168)$ & 0.294 \\
\hline Nosocomial bacteremia & $568(89.9)$ & $153(86.4)$ & $0.776(0.424-1.420)$ & 0.195 \\
\hline 72h-IIAT & $158(25.0)$ & $68(38.4)$ & $1.872(1.316-2.662)$ & $<0.001$ \\
\hline \multicolumn{5}{|l|}{ Patterns of pathogen resistance } \\
\hline MDR bacteria & $429(67.9)$ & $116(65.5)$ & $0.900(0.633-1.280)$ & 0.557 \\
\hline CR-GNB & $40(6.3)$ & $38(21.5)$ & $4.046(2.501-6.545)$ & $<0.001$ \\
\hline \multicolumn{5}{|l|}{ Laboratory parameters } \\
\hline Hemoglobin $<70 \mathrm{~g} / \mathrm{dL}$ & $527(83.4)$ & $136(76.8)$ & $0.66 I(0.440-0.993)$ & 0.045 \\
\hline Platelet $<10 \times 10^{3} \mathrm{~mm}^{-3}$ & $403(63.8)$ & $105(59.3)$ & $0.829(0.589-1.165)$ & 0.280 \\
\hline Albumin $<30 g / L$ & $339(53.6)$ & $93(52.5)$ & $0.957(0.685-1.336)$ & 0.796 \\
\hline AST $>120 U / L$ & $63(10.0)$ & I4(7.9) & $0.776(0.424-1.420)$ & 0.409 \\
\hline $\mathrm{TBil}>34.2 \mu \mathrm{mol} / \mathrm{L}$ & $84(\mid 3.3)$ & $26(14.7)$ & $1.123(0.698-1.807)$ & 0.631 \\
\hline $\mathrm{PT}>14 \mathrm{~s}$ & $157(24.8)$ & $4 I(23.2)$ & $0.912(0.616-1.351)$ & 0.646 \\
\hline 7-day mortality & $61(9.7)$ & $40(22.6)$ & $2.733(1.760-4.244)$ & $<0.001$ \\
\hline
\end{tabular}

Abbreviations: CVC, centre vein catheter; 72h-IIAT, 72h-initial inappropriate antibiotic treatment; CR-GNB, carbapenem-resistance gram negative bacteria; MDR bacteria, multidrug resistance bacteria; AST, aspartate transaminase; TBil, total bilirubin; PT, prothrombin time.

of invasive operations and initial treatment of appropriate antibiotics.

The influence of different antibiotic resistance mechanisms on the prognosis of patients with BSI has been a highly debated topic. ${ }^{1,4-6,9,21,28,29}$ An Italian study investigating HM patients with Pseudomonas aeruginosa BSI reported that the 21-day mortality of patients infected with MDR bacteria was significantly worse than that of patients infected with non-MDR bacteria $(42.4 \%$ vs $12.5 \%, p=0.03) .{ }^{28}$ However, another study conducted by Scheich et al reported that the two kinds 

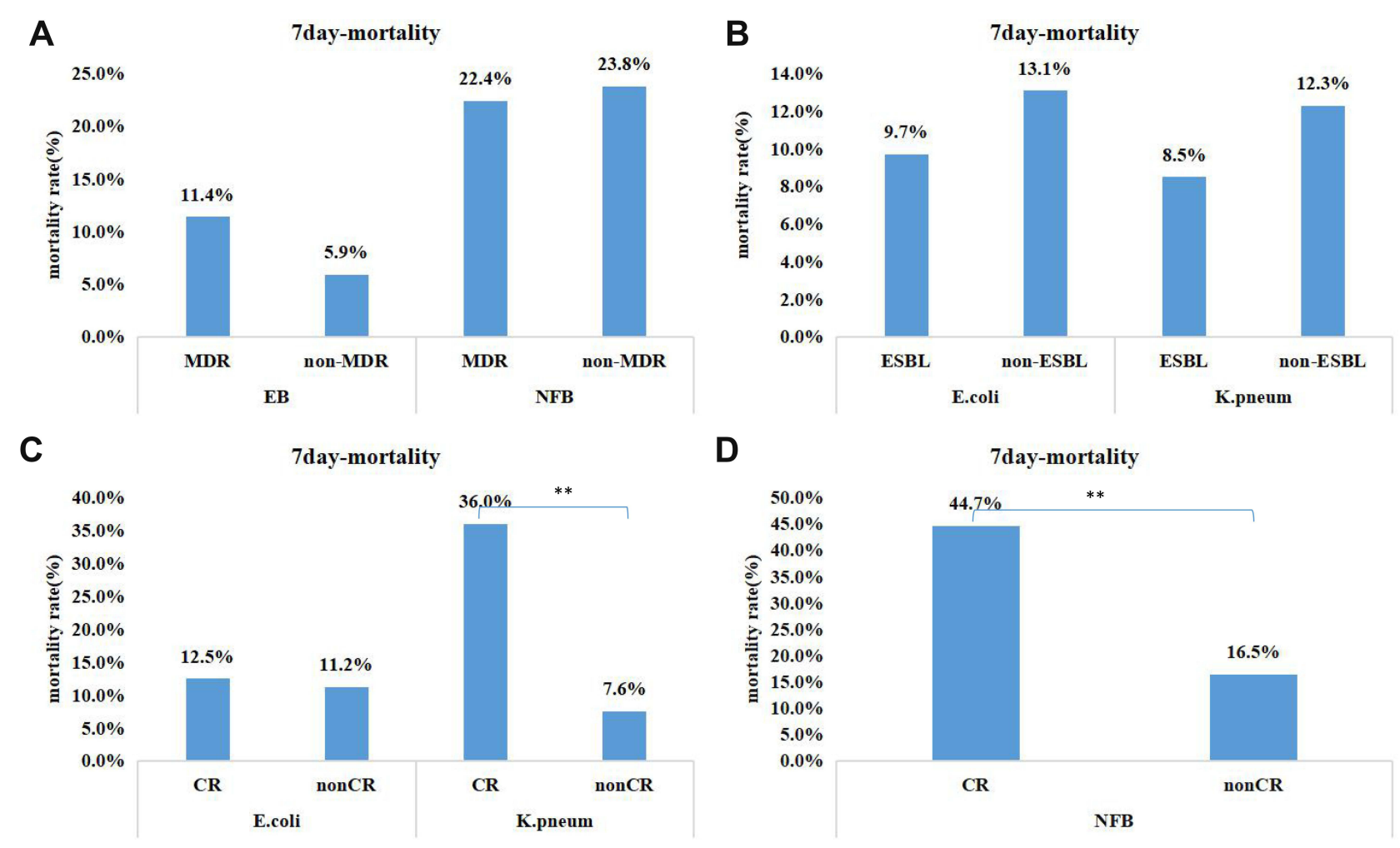

Figure 3 Impact of pathogen antibiotic resistance profile on 7 day-mortality of patients with BSI. (A) Multidrug resistance on prognosis of Enterobacteriaceae and Nonfermentative bacteria; (B) ESBL production on prognosis of Escherichia coli and Klebsiella pneumoniae; (C) Carbapenem resistance on prognosis of Escherichia coli and Klebsiella pneumoniae; (D) Carbapenem resistance on prognosis of Non fermenting bacteria. $* * \mathrm{P}<0.00 \mathrm{I}$.

Abbreviations: $\mathrm{EB}$, Enterobacteriaceae; NFB, non fermentative bacteria; MDR, multi-drug resistance; ESBL, extended-spectrum $\beta$-lactamase; CR, carbapenem-resistant; $E$. coli, Escherichia coli; K. pneum, Klebsiella pneumoniae.

of antibiotic resistance mechanisms had an equivalent effect on patient outcomes. ${ }^{5}$ Our results showed that although the early mortality of patients with HMs who had Enterobacter MDR BSI is higher compared to patients with non-MDR bacteria BSI, the difference was not statistically significant. This may be related to the initial use of appropriate antibiotics in more than $75 \%$ of patients (Table 3). Additionally, whether ESBL affects the prognosis of patients is also debatable A South Korean study showed that pathogens with ESBL production could impair the prognosis of patients with $\mathrm{HMs},{ }^{29}$ while other trials have indicated conflicting results. ${ }^{12,30}$ The results of our study showed that ESBL production of E. coli and $K$. pneumoniae had little effect on the early mortality of patients $(\mathrm{P}>0.05)$. However, these contradictory results may be a result of insufficient sample size and different initial medications. Of note, the majority of studies have consistently reported that the existence of carbapenem resistance in bacteria could significantly impact the prognosis of patients. ${ }^{18,26,31,32}$ Compared with carbapenem-sensitive bacteria-related BSI, patients who were infected with carbapenem-resistant bacteria had more unfavorable outcomes. CR- Klebsiella Pneumoniae infected patients were particularly vulnerable, which may be due to the highly resistant features of CR- Klebsiella Pneumoniae against common antibiotics. Our study draws the same conclusion that infections related to CR-Klebsiella Pneumoniae puts patients with HMs at greater disadvantage. Given the huge impact of CR bacteria on the prognosis of patients, antibiotic prophylaxis and decolonization should be considered for patients who are at high-risk for infection and have positive CRE screening.

To our knowledge, this is the first study to investigate the influence of bacteriological factors on the prognosis of patients with HMs who had BSI. However, this study has several limitations. First, as a retrospective research, we could not obtain bacterial samples for homology analysis and determine the distribution of drug-resistant genotypes, it cannot be analyzed from a deeper level, while the genotypes of E. coli in China are mostly NDM, while those of Klebsiella pneumoniae are mostly $\mathrm{KPC}^{33}$ Second, our study relied on inpatient records, and we could only analyze objective and easily measurable outcomes, such as patients' all-cause 7-day mortality. More well-designed prospective studies based on bacterial genotypes are needed in the future. 


\section{Conclusion}

In conclusion, GNB antibiotic resistance, particularly CRGNB, has become an increasingly notable problem for patients with HMs. Both pathogen type and patterns of antibiotic resistance can affect the early outcome of patients. Clinical attention should be paid in particular to infections related to non-fermentative bacteria and carbapenem-resistant bacteria.

\section{Ethical Statement}

This study was approved by the ethics committee of the Third Xiangya Hospital, Central South. Our study was a retrospective study, this study would not do harm to rights, benefits, and health of the subjects, no personally identifiable information was collected in this study. So the requirement for informed consent from patients was also waived, and we guarantee the covering patient data confidentiality. This study was conducted in accordance with the Declaration of Helsinki.

\section{Acknowledgments}

We thank all those who helped us in this study, in particular, the Department of Hematology and the Department of Clinical Laboratory for making this study possible.

\section{Author Contributions}

All authors meet the ICMJE authorship criteria. All authors made substantial contributions to the work, Xin Li for conception and design, Yishu Tang for analysis and interpretation of data and took part in drafting the article, Cong Xu, Han Xiao, Qian Cheng acquisition of data and Liwen Wang for checking the English version of the manuscript. All authors made a significant contribution to the work reported, whether that is in the conception, study design, execution, acquisition of data, analysis and interpretation, or in all these areas; took part in drafting, revising or critically reviewing the article; gave final approval of the version to be published; have agreed on the journal to which the article has been submitted; and agree to be accountable for all aspects of the work.

\section{Funding}

This work was supported by the National Natural Science Foundation of China (General Program), N0. 81870165 and Special project for the construction of innovatiove provinces in Hunan, No. 2020SK53619 and 2021JJ31013. We thank Francis Yang for checking the English version of the manuscript.

\section{Disclosure}

The authors declare that they have no conflicts of interest in this work.

\section{References}

1. Chen CY, Tsay W, Tang JL, et al. Epidemiology of bloodstream infections in patients with haematological malignancies with and without neutropenia. Epidemiol Infect. 2010;138(7):1044-1 051.

2. Gudiol C, Bodro M, Simonetti A, et al. Changing aetiology, clinical features, antimicrobial resistance, and outcomes of bloodstream infection in neutropenic cancer patients. Clin Microbiol Infect. 2013;19(5):474-479. doi:10.1111/j.1469-0691.2012.03879.x

3. Trecarichi EM, Pagano L, Candoni A, et al. Current epidemiology and antimicrobial resistance data for bacterial bloodstream infections in patients with hematologic malignancies: an Italian multicentre prospective survey. Clin Microbiol Infect. 2015;21(4):337-343. doi:10.1016/j.cmi.2014.11.022

4. Zhu J, Zhou K, Jiang Y, et al. Bacterial pathogens differed between neutropenic and non-neutropenic patients in the same hematological ward: an 8-year survey. Clin Infect Dis. 2018;67(suppl_2):S174S178. doi:10.1093/cid/ciy643

5. Scheich S, Weber S, Reinheimer C, et al. Bloodstream infections with gram-negative organisms and the impact of multidrug resistance in patients with hematological malignancies. Ann Hematol. 2018;97 (11):2225-2234.

6. Averbuch D, Orasch C, Cordonnier C, et al. European guidelines for empirical antibacterial therapy for febrile neutropenic patients in the era of growing resistance: summary of the 2011 4th European conference on infections in leukemia. Haematologica. 2013;98 (12):1826-1835. doi:10.3324/haematol.2013.091025

7. Mert D, Ceken S, Iskender G, et al. Epidemiology and mortality in bacterial bloodstream infections in patients with hematologic malignancies. J Infect Dev Ctries. 2019;13(8):727-735. doi:10.3855/ jidc. 11457

8. Ruhnke M, Arnold R, Gastmeier P. Infection control issues in patients with haematological malignancies in the era of multidrug-resistant bacteria. Lancet Oncol. 2014;15(13):e606-e619. doi:10.1016/S1470-2045(14)70344-4

9. Magiorakos AP, Srinivasan A, Carey RB, et al. Multidrug-resistant, extensively drug-resistant and pandrug-resistant bacteria: an international expert proposal for interim standard definitions for acquired resistance. Clin Microbiol Infect. 2012;18(3):268-281.

10. Satlin MJ, Walsh TJ. Multidrug-resistant Enterobacteriaceae, Pseudomonas aeruginosa, and vancomycin-resistant Enterococcus: three major threats to hematopoietic stem cell transplant recipients. Transpl Infect Dis. 2017;19(6):e12762. doi:10.1111/tid.12762

11. Gudiol C, Calatayud L, Garcia-Vidal C, et al. Bacteraemia due to extended-spectrum beta-lactamase-producing Escherichia coli (ESBL-EC) in cancer patients: clinical features, risk factors, molecular epidemiology and outcome. J Antimicrob Chemother. 2010;65 (2):333-341. doi:10.1093/jac/dkp411

12. Kang CI, Chung DR, Ko KS, Peck KR, Song JH. Risk factors for infection and treatment outcome of extended-spectrum beta-lactamase-producing Escherichia coli and Klebsiella pneumoniae bacteremia in patients with hematologic malignancy. Ann Hematol. 2012;91(1):115-121. doi:10.1007/s00277-011-12 47-7

13. Cornejo-Juarez P, Vilar-Compte D, Perez-Jimenez C, NamendysSilva SA, Sandoval-Hernandez S, Volkow-Fernandez P. The impact of hospital-acquired infections with multidrug-resistant bacteria in an oncology intensive care unit. Int J Infect Dis. 2015;31:31-34. doi:10.1016/j.ijid.2014.12.022 
14. Kim SH, Kwon JC, Choi SM, et al. Escherichia coli and Klebsiella pneumoniae bacteremia in patients with neutropenic fever: factors associated with extended-spectrum beta-lactamase production and its impact on outcome. Ann Hematol. 2013;92(4):533-541. doi:10.1007/ s00277-012-1631-y

15. Chinese Society of Hematology CM, Chinese Medical Doctor Association. [Chinese guidelines for the clinical application of antibacterial drugs for agranulocytosis with fever (2016)]. Zhonghua Xue Ye Xue Za Zhi. 2016;37(5):353-359. [Chinese]

16. Genga KR, Russell JA. Update of sepsis in the intensive care unit. J Innate Immun. 2017;9(5):441-455. doi:10.1159/000477419

17. Ghafur A, Devarajan V, Raja T, et al. Monotherapy versus combination therapy against nonbacteremic carbapenem-resistant gram-negative infections: a retrospective observational study. Indian J Crit Care Med. 2017;21 (12):825-829. doi:10.4103/ijccm.IJCCM_243_17

18. Tamma PD, Goodman KE, Harris AD, et al. Comparing the outcomes of patients with carbapenemase-producing and non-carbapenemaseproducing carbapenem-resistant Enterobacteriaceae bacteremia. Clin Infect Dis. 2017;64(3):257-264. doi:10.1093/cid/ciw741

19. Tang $\mathrm{Y}, \mathrm{Wu} \mathrm{X}, \mathrm{Cheng} \mathrm{Q}, \mathrm{Li} \mathrm{X}$. Inappropriate initial antimicrobial therapy for hematological malignancies patients with gram-negative bloodstream infections. Infection. 2020;48(1):109-116. doi:10.1007/ s15010-019-01370-X

20. Tang Y, Cheng Q, Yang Q, et al. Prognostic factors and scoring model of hematological malignancies patients with bloodstream infections. Infection. 2018;46(4):513-521. doi:10.1007/s15010-0181151-3

21. Zilberberg MD, Shorr AF, Micek ST, Vazquez-Guillamet C, Kollef MH. Multi-drug resistance, inappropriate initial antibiotic therapy and mortality in gram-negative severe sepsis and septic shock: a retrospective cohort study. Crit Care. 2014;18(6):596. doi:10.1186/s13054-014-0596-8

22. Marin M, Gudiol C, Ardanuy C, et al. Bloodstream infections in neutropenic patients with cancer: differences between patients with haematological malignancies and solid tumours. J Infect. 2014;69 (5):417-423. doi:10.1016/j.jinf.2014.05.018

23. Zhai W, Zhang X, Wei J, et al. A prospective observational study of antibiotic therapy in febrile neutropenia patients with hematological malignances from multiple centers in Northeast China. Int $J$ Infect Dis. 2015;37:97-103. doi:10.1016/j.ijid.2015.04.015

24. Pagano L, Caira M, Trecarichi EM, et al. Carbapenemase-producing Klebsiella pneumoniae and hematologic malignancies. Emerg Infect Dis. 2014;20(7):1235-1236. doi:10.3201/eid2007.130094
25. Hu F, Zhu D, Wang F, Wang M. Current status and trends of antibacterial resistance in China. Clin Infect Dis. 2018;67(suppl_2): S128-34. doi:10.1093/cid/ciy657

26. Liu J, Wang H, Huang Z, et al. Risk factors and outcomes for carbapenem-resistant Klebsiella pneumoniae bacteremia in onco-hematological patients. J Infect Dev Ctries. 2019;13 (5):357-364. doi:10.3855/jidc. 11189

27. Al-Anazi KA, Al-Jasser AM. Infections caused by Acinetobacter baumannii in recipients of hematopoietic stem cell transplantation. Front Oncol. 2014;4:186.

28. Trecarichi EM, Tumbarello M, Caira M, et al. Multidrug resistant Pseudomonas aeruginosa bloodstream infection in adult patients with hematologic malignancies. Haematologica. 2011;96(1):e1-e3, e4. doi:10.3324/haematol.2010.036640

29. Ha YE, Kang CI, Cha MK, et al. Epidemiology and clinical outcomes of bloodstream infections caused by extended-spectrum beta-lactamase-producing Escherichia coli in patients with cancer. Int $J$ Antimicrob Agents. 2013;42(5):403-409. doi:10.1016/j. ijantimicag.2013.07.018

30. Liang T, Xu C, Cheng Q, Tang Y, Zeng H, Li X. Epidemiology, risk factors, and clinical outcomes of bloodstream infection due to extended-spectrum beta-lactamase-producing Escherichia coli and Klebsiella pneumoniae in hematologic malignancy: a retrospective study from Central South China. Microb Drug Resist. 2020;27 (6):800-808

31. Zhang $\mathrm{P}$, Wang J, Hu H, et al. Clinical Characteristics and risk factors for bloodstream infection due to carbapenem-resistant Klebsiella pneumoniae in patients with hematologic malignancies. Infect Drug Resist. 2020;13:3233-3242. doi:10.2147/IDR.S272217

32. Satlin MJ, Cohen N, Ma KC, et al. Bacteremia due to carbapenem-resistant Enterobacteriaceae in neutropenic patients with hematologic malignancies. $J$ Infect. 2016;73(4):336-345. doi:10.1016/j.jinf.2016.07.002

33. Zhang Y, Wang Q, Yin Y, et al. Epidemiology of carbapenem-resistant enterobacteriaceae infections: report from the China CRE network. Antimicrob Agents Chemother. 2018;62(2). doi:10.1128/AAC.01882-17.

34. Zhao Y, Lin Q, Liu L, et al. Risk factors and outcomes of antibiotic-resistant pseudomonas aeruginosa bloodstream infection in adult patients with acute leukemia. Clin Infect Dis. 2020;71 (Suppl 4):S386-S393. doi:10.1093/cid/ciaa1522
Infection and Drug Resistance

\section{Publish your work in this journal}

Infection and Drug Resistance is an international, peer-reviewed openaccess journal that focuses on the optimal treatment of infection (bacterial, fungal and viral) and the development and institution of preventive strategies to minimize the development and spread of resistance. The journal is specifically concerned with the epidemiology of antibiotic resistance and the mechanisms of resistance development and diffusion in both hospitals and the community. The manuscript management system is completely online and includes a very quick and fair peerreview system, which is all easy to use. Visit http://www.dovepress.com/ testimonials.php to read real quotes from published authors. 